\title{
Time Dose Relation of Caffeine Ingestion on Muscular Strength and Endurance Performance
}

\author{
Jaspal S Sandhu, Shweta Shenoy, I Dutt
}

\begin{abstract}
Background: There have been numerous studies and reviews indicating that caffeine ingested before exercise causes rapid and significant improvement in performance, especially in aerobic capacity. Even though caffeine has a half life of 4 to 6 hours that implies high level of caffeine will be in the blood for up to 3 to 4 hours after ingestion, most studies on exercise performance are at 1 hour after ingestion.
\end{abstract}

Objectives: To assess the efficacy of $300 \mathrm{mg}$ of caffeine and placebo ingestion at 1 hour, 1 hour 30 minutes, 2 hours, 2 hours 30 minutes, 3 hours in improvement of isometric muscle strength in human quadriceps muscle, cardiorespiratory endurance and to find that which of the duration produce best result.

Materials and methods: Experimental study with different subject design. Twenty subjects with equal number of boys and girl (age 20-30 years, weight 60-65 kg) were recruited to participate in the study. They were randomly allocated to experimental and placebo group. Pre- and postquadriceps muscle strength and time to exhaustion at $65 \%$ of $\mathrm{VO}_{2 \max }$ was recorded.

Results: The results showed significant improvement $(p<0.001)$ in strength and endurance at all five durations in experimental group. Results were significant $(p<0.05)$ when caffeine and placebo groups were compared. The percentage improvement for strength and endurance was higher at 1st and 2nd hours when compared to 3rd hours duration.

Conclusion: The effects of caffeine are highest till 2 hours of ingestion after that wean off effects are seen.

Keywords: Caffeine, Strength, Endurance.

How to cite this article: Sandhu JS, Shenoy S, Dutt I. Time Dose Relation of Caffeine Ingestion on Muscular Strength and Endurance Performance. J Postgrad Med Edu Res 2012; 46(1):19-23.

\section{Source of support: Nil}

Conflict of interest: None declared

\section{INTRODUCTION}

Caffeine in one of the most widely consumed drugs in the world and is known to be ingested by sports people to augment performance. Laboratory-based ${ }^{1-7}$ and some field based $^{8,9}$ researches demonstrate the beneficial effects of caffeine on endurance exercise performance. Mechanism of action that may explain this ergogenic effect is an increase in free fatty acid (FFA) oxidation and a subsequent sparing of muscle glycogen. ${ }^{10}$ Caffeine exerts this effect through competitive antagonism of adenosine receptors at physiological concentration. ${ }^{11}$
There is substantive evidence that caffeine facilitates neuromuscular function at the level of the sarcoplasmic reticulum (SR). The potentiation of muscle contractile force has also been demonstrated at concentrations that are attainable with ergogenic doses of caffeine in humans.

Even though caffeine has half life of 4 to 6 hours high levels of caffeine will be in blood for up to 3 to 4 hours ingestion. The majority of laboratory studies demonstrating the ergogenic effect of caffeine ingestion on performance administer caffeine dose 1 hour before exercise. The assumption is that the ergogenic effect is related to the circulating level of drug in the blood. Thus, maximal effects are assumed to occur $\sim 1$ hour after ingestion when peak plasma concentration is observed. ${ }^{12,13}$ However, it is unknown that this is the optimal timing of caffeine administration to maximize its ergogenic effects. Some studies ${ }^{14,15}$ have suggested that waiting for 3 hours may be more optimal because of caffeine-induced effect on lipolysis is greater than at earlier time after ingestion. However, the hypothesis that the ergogenic effect from caffeine is due to an enhanced FFA mobilization and tissue utilization has not found much support in the recent literature. ${ }^{16}$

Thus, the purpose of this study was to assess the efficacy of drug at duration 1 hour, 1 hour 30 minutes, 2 hours, 2 hours 30 minutes and 3 hours on muscle strength and cardiorespiratory endurance after ingestion.

\section{MATERIALS AND METHODS}

An experimental study with different subject design was conducted.

A group of 20 (10 males, 10 females) healthy adults with mean age $22 \pm 5$ years and mean weight $60 \pm 5 \mathrm{~kg}$ were included in the study. The subjects were asked to complete questionnaire concerning their daily caffeine intake. Total caffeine intake per day was not more than 2 to 3 cups of tea which is equivalent to $200 \mathrm{mg}$ of caffeine. The experimental protocol and potential risks of the study were explained to each subject both verbally and in writing and their informed consent was obtained. The study was approved by Institutional Ethical Committee at GNDU, Amritsar.

\section{Preexperimental Protocol Assessment}

Strength assessment by isoinertial dynamometer: It represents reliable means to access muscle strength. Each 
subject was made to sit on the isoinertial dynamometer and perform maximum voluntary contraction with 10 seconds hold on the dynamometer to determine the peak force and average force of the quadriceps muscle of the dominant leg (at $60^{\circ} \mathrm{C}$ of knee flexion). These constituted the baseline reading of experimental and placebo group.

Cardiorespiratory endurance assessment by vista turbo trainer: Each subject performed an incremental $\mathrm{VO}_{2 \max }$ test (Ramp 20 Protocol on an electromagnetically braked cycle ergometer) to determine the maximal power output (wattage maximum, $\left.\mathrm{W}_{\max }\right)$ and maximal oxygen uptake $\left(\mathrm{VO}_{2 \max }\right)$. Subsequently, the subject pedaled at work load of $65 \%$ of $\mathrm{VO}_{2 \max }$ until exhaustion (when pedaling frequency was lower than 50 revolutions per minute) and time to exhaustion was recorded.

\section{Experimental Protocol}

Drug trial was scheduled once a week. Subjects ingested either placebo or standardized caffeine capsules (300 mg) depending upon the group to which they belonged to (experimental and placebo) and arrived 1 hour, 1 hour 30 minutes, 2 hours, 2 hours 30 minutes and 3 hours before exhaustion ride and strength testing (peak force and average force) according to the duration trial which was scheduled for the day.

\section{STATISTICAL ANALYSIS}

Data was presented as Mean \pm SD. The data was analyzed for statistical significance by using the statistical package for social sciences (SPSS 14.0 software). To assess the changes in strength and cardiorespiratory endurance at different durations related t-test was used. Unrelated t-test was used to compare experimental group with placebo group.

\section{RESULTS}

Peak force: On intragroup comparison of peak force in pre- and postcaffeine ingestion at different durations, it was found that peak force increased significantly at all durations $(p<0.001)$ (Fig. 1). On intergroup comparison of peak force in experimental and placebo group at different durations, it was found that peak force increased significantly in experimental group $(\mathrm{p}<0.05)$ (Fig. 2). On intragroup comparison of peak force at durations 1 to 2, 1 to 3 and 2 to 3 the results were nonsignificant. Percentage increase in peak force at 1st hour was $18.85 \%$, 2nd hour was $19.40 \%$ and 3rd hour was $13.13 \%$ (Fig. 3).

Average force: On intragroup comparison of average force in pre- and postcaffeine ingestion at different durations, it was found that average force increased significantly ( $p<0.001$ ) all durations (Fig. 4). On intergroup comparison of average force in experimental and placebo group at

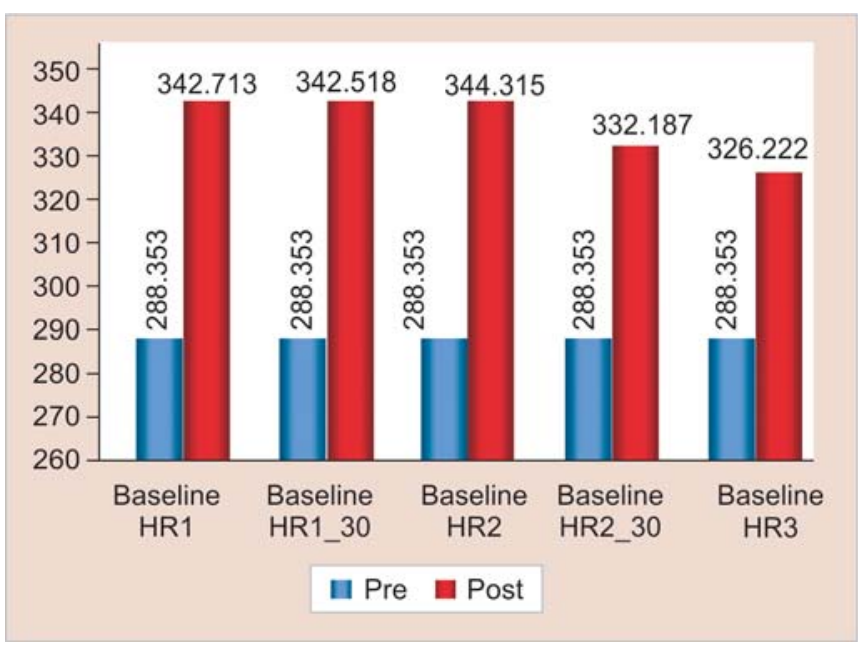

Fig. 1: Distribution of mean value of peak force before and after caffeine intake at all five durations

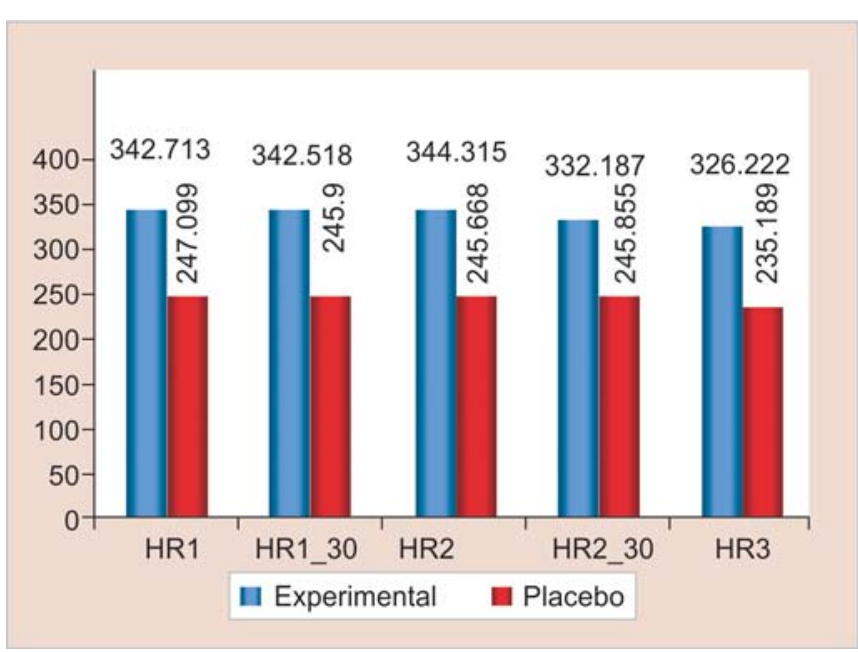

Fig. 2: Distribution of mean values of peak force in experimental group and placebo group at all five durations

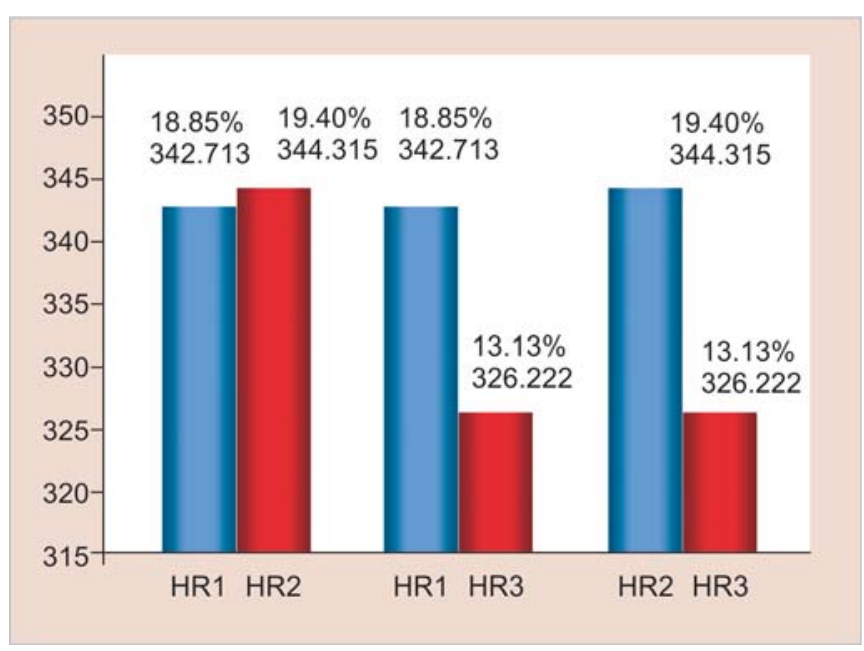

Fig. 3: Distribution of mean values of peak force at durations 1 to 2,1 to 3 and 2 to 3 in experimental group 
different durations, it was found that average force increased significantly in experimental group ( $<<0.10)$ (Fig. 5). On intragroup comparison of average force at durations 1 to 2 , 1 to 3 and 2 to 3 the results were nonsignificant. Percentage increase in average force at 1st hour was $19.76 \%$, 2nd hour was $22.45 \%$ and 3rd hour was $16.31 \%$ (Fig. 6).

Time to exhaustion: On intragroup comparison of time to exhaustion in pre- and postcaffeine ingestion at different durations, it was found that time to exhaustion increased significantly $(\mathrm{p}<0.001)$ at all durations (Fig. 7). On intergroup comparison of time to exhaustion in experimental and placebo group at different durations, it was found that time to exhaustion increased significantly in experimental group ( $\mathrm{p}<0.10$ ) at all durations (Fig. 8). On intragroup comparison of time to exhaustion at durations 1 to 2,1 to 3 and 2 to 3 the results were nonsignificant. Percentage increase in average force at 1st hour was $20.22 \%$, 2nd hour was $19.80 \%$ and 3rd hour was $18.81 \%$ (Fig. 9).

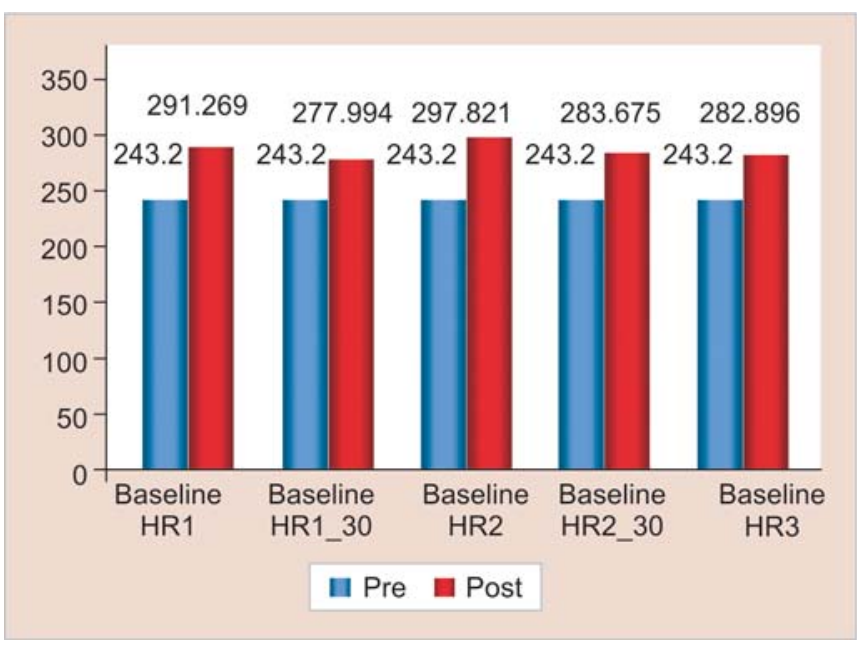

Fig. 4: Distribution of average force before and after caffeine intake at all five durations

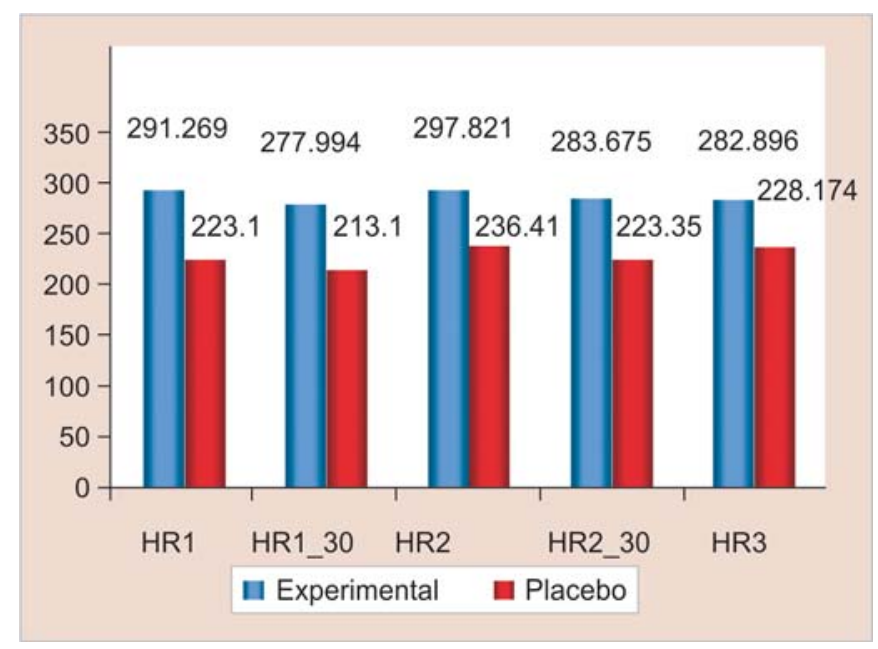

Fig. 5: Distribution of mean values of average force in experimental group and placebo group at all five durations

\section{DISCUSSION}

The results showed significant increase in peak force, average force and time to exhaustion at all durations. The percentage increase for peak force, average force and time to exhaustion was highest in 1st hour and 2nd hour duration when compared with 3rd hour.

The results of present study in the improvement of muscle strength are in agreement with following studies. Study conducted by Beck TW (2006) ${ }^{17}$ reported statistically significant increase in free weight bench press (BP) exercise post 1 hour in group which was administered caffeine.

Furthermore, James and Wilson (2005) $)^{18}$ investigated the effects of $70 \mu \mathrm{m}$ caffeine on force and power output in isolated mouse extensor digitorum longus muscle in vitro and reported a significant increase (2-3\%) in peak force and net work produced during work loops; suggesting that caffeine ingestion enhance force and power during brief but not prolonged activities. The significant increase in peak

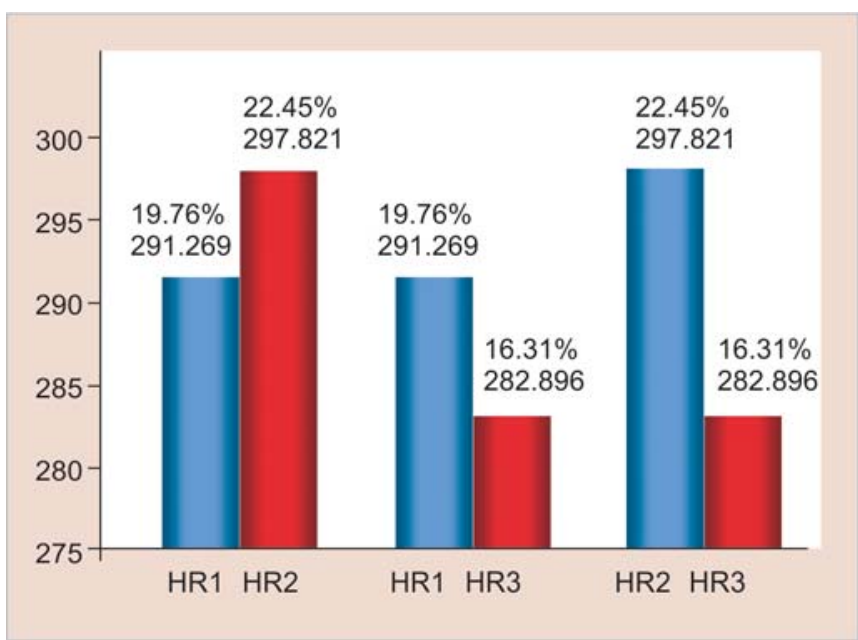

Fig. 6: Distribution of mean values of average force at durations 1 to 2,1 to 3 and 2 to 3 in experimental group

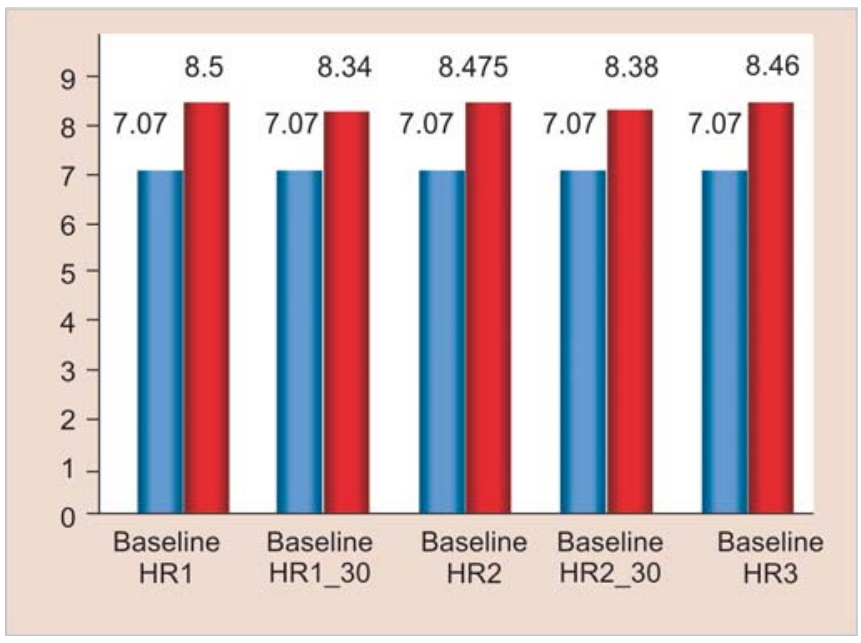

Fig. 7: Distribution of time till exhaustion before and after caffeine intake at all five durations 


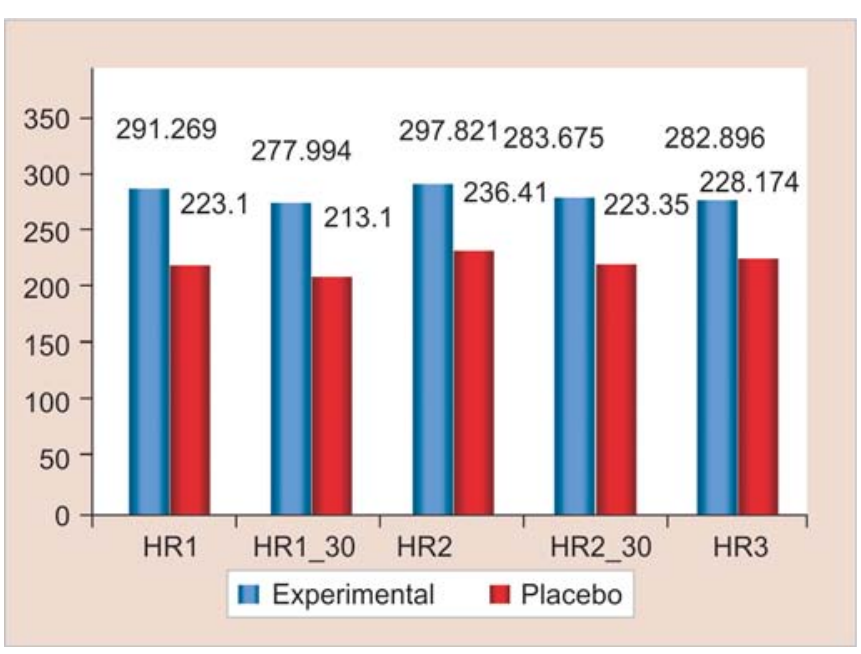

Fig. 8: Distribution of mean values of time till exhaustion in experimental group and placebo group at all five durations

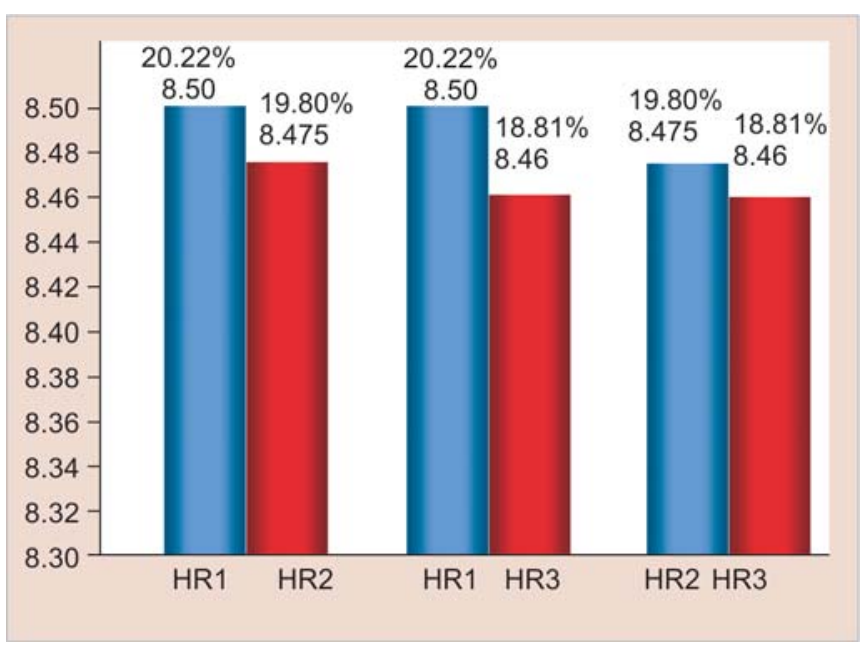

Fig. 9: Distribution of mean values of time till exhaustion at durations 1 to 2,1 to 3 and 2 to 3 in experimental group

muscle force can be attributed to following mechanisms, (a) caffeine mobilizes and increase release of intracellular $\mathrm{Ca}^{2+}$ from sarcoplasmic reticulum, ${ }^{19,20}$ (b) inhibition of phosphodiesterase by caffeine leading to a subsequent increase in cyclic 3'5' adenosine mophosphate (camp) in various body tissues.

In the present study, caffeine ingestion led to increase in cardiorespiratory endurance at all durations. These findings are consistant with the findings of Bell DG and Mcllean TM (2002) ${ }^{21}$ who studied exercise endurance 1, 3, 6 hours after caffeine ingestion in caffeine users and nonusers and reported an increase in endurance at all three durations. Another study by Pasman WJ and Van Beak MA $(1995)^{22}$ also reported an increase in endurance performance time after ingesting 5, 9, $13 \mathrm{mg} / \mathrm{kg}$ body weight of caffeine. No difference was found in endurance performance between the 3 caffeine dosages which indicate no dose-response relation of caffeine and endurance.
Improvement in performance after absorption of caffeine observed in prolonged exercise involving aerobic metabolism can be explained by the stimulation of lipolysis. ${ }^{23}$ The lipolysis might be increased via direct or indirect effect. Furthermore, the increase in endurance performance after caffeine ingestion is thought to be caused by enhanced fat oxidation which delays glycogen depletion and fatigue. ${ }^{24,25}$

The findings of the present study that maximum effects of caffeine are observed in the first 2 hours can be explained by the fact that the peak plasma concentration which is attained 1 hour after caffeine ingestion.

In light of this study, it can be stated that there was significant increase in strength and endurance at all durations. But the percentage improvement was higher at 1st and 2nd hours duration when compared with the 3rd hour duration. Henceforth, it can be concluded that maximum effects caffeine are observed in first 2 hours after ingestion after that wean off effects are seen.

\section{ACKNOWLEDGMENT}

We acknowledge Sigma Aldrich Chemicals Pvt Ltd for providing the caffeine.

\section{REFERENCES}

1. Costill DL, Dalsky GP, Fink WJ. Effects of caffeine ingestion on metabolism and exercise performance. Med Sci Sports 1978;10:155-58.

2. Graham TE, Hibbert E, Sathasivam P. Metabolic and exercise endurance effects of coffee and caffeine ingestion. J Appl Physiol 1998;85:883-89.

3. Graham TE, Spriet LL. Performance and metabolic responses to a high caffeine dose during prolonged exercise. J Appl Physiol 1991;71:2292-98.

4. Graham TE, Spriet LL. Metabolic, catecholamine, and exercise performance responses to various doses of caffeine. J Appl Physiol 1995;78:867-74.

5. Ivy JL, Costill DL, Fink WJ, Lower RW. Influence of caffeine and carbohydrate feedings on endurance performance. Med Sci Sports 1979;11:6-11.

6. Kovacs EM, Stegen J, Brouns F. Effect of caffeinated drinks on substrate metabolism, caffeine excretion and performance. J Appl Physiol 1998;85:709-15.

7. Pasman WJ, van Baak MA, Jeukendrup AE, de Haan A. The effect of different dosages of caffeine on endurance performance time. Int J Sports Med 1995;16:225-30.

8. Berglund B, Hemmingsson P. Effects of caffeine ingestion on exercise performance at low and high altitudes in cross-country skiers. Int J Sports Med 1982;3:234-36.

9. MacIntosh BR, Wright BM. Caffeine ingestion and performance of a 1,500-metre swim. Can J Appl Physiol 1995;20: 168-77.

10. Mark T, Cynthia C. Caffeine potentiates low frequency skeletal muscle force in habitual and nonhabitual caffeine consumers. J of Appl Physiol 2000;89:1719-24. 
11. Fryer MW, Neering IR. Actions of caffeine on fast- and slowtwitch muscles of the rat. J Physiol (Lond) 1998;416:435-54.

12. Bonati M, Latini R, Galletti F, Young JF, Tognoni G, Garattini S. Caffeine disposition after oral doses. Clin Pharmacol Ther 1982;32:98-106.

13. Fredholm BB, Battig K, Holmen J, Nehlig A, Zvartau EE. Actions of caffeine in the brain with special reference to factors that contribute to its widespread use. Pharmacol Rev 1999;51: 83-133.

14. Nehlig A, Debry G. Caffeine and sports activity: A review. Int J Sports Med 1994;15:215-23.

15. Weir J, Noakes TD, Myburgh K, Adams B. A high carbohydrate diet negates the metabolic effects of caffeine during exercise. Med Sci Sports Exerc 1987;19:100-05.

16. Graham TE. Caffeine and exercise: Metabolism, endurance and performance. Sports Med 2001;31:785-807.

17. Beck TW. The acute effects of caffeine containing supplements on strength, muscular endurance and anaerobic capabilities. J Strength and Conditioning Research 2006;20:506-10.

18. James RS, Wilson RS. Effects of caffeine on mouse skeletal muscle power output during recovery from fatigue. J App Physiol 2004;96:545-52.

19. Block BM, Barry SR, Faulkner JA. Aminophylline increases submaximum power but not intrinsic velocity of shortening of frog muscle. J Appl Physiol 1992;73:71-74.

20. Lopes JM, Jardim AJ, Aranda JV, Macklem PT. Effect of caffeine on skeletal muscle function before and after fatigue. J Appl Physiol 1983;54:1303-05.

21. Bell DG, Mclellan TM. Exercise endurance 1, 3, 6 hours after caffeine ingestion in caffeine users and nonusers. J Appl Physiol 2002;93:1227-37.
22. Pasman WJ, Vaanbaak MA, Jeukendrup AE, Han AD. The effects of different dosages of caffeine on endurance. Int J Sports Med 1995;16:225-30.

23. Essig D, Costill DL, van Handel PJ. Effects of caffeine ingestion on utilization of muscle glycogen and lipid during leg ergometer cycling. Int J Sports Med 1980;1:86-90.

24. Erickson MA, Schwarzkopf RJ, McKenzie RD. Effects of caffeine, fructose, and glucose ingestion on muscle glycogen utilization during exercise. Med Sci Sports Exerc 1987;19: 579-83.

25. Spriet LL, MacLean DA, Dyck DJ, Hultman E, Cederblad G, Graham TE. Caffeine ingestion and muscle metabolism during prolonged exercise in humans. Am J Physiol Endocrinol Metab 1992;262:E891-98.

\section{ABOUT THE AUTHORS}

\section{Jaspal S Sandhu (Corresponding Author)}

Dean, Professor, Faculty of Sports Medicine and Physiotherapy Guru Nanak Dev University, Amritsar, Punjab, India e-mail: jassandhu2000@yahoo.com.in

\section{Shweta Shenoy}

Reader, Department of Sports Medicine and Physiotherapy, Guru Nanak Dev University, Amritsar, Punjab, India

\section{Dutt}

Department of Sports Medicine and Physiotherapy, Guru Nanak Dev University, Amritsar, Punjab, India 